\title{
Affective and Perceptual Responses during Reduced-Exertion High-Intensity Interval Training (REHIT)
}

\author{
Preeyaphorn Songsorn ${ }^{1}$, Noel Brick ${ }^{2}$, Ben Fitzpatrick ${ }^{3}$, Sinead Fitzpatrick ${ }^{4}$, Gary \\ McDermott $^{5}$, Conor McClean ${ }^{3}$, Gareth W. Davison ${ }^{3}$, Niels B.J. Vollaard ${ }^{1} \&$ Richard S. $^{2}$ \\ Metcalfe ${ }^{6 *}$
}

${ }^{1}$ Faculty of Health Sciences and Sport, University of Stirling, Stirling, UK

${ }^{2}$ Psychology Research Institute, Ulster University, Northern Ireland, UK

${ }^{3}$ Sport and Exercise Science Research Institute, Ulster University, Northern Ireland, UK

${ }^{4}$ Western Health and Social Care Trust, Altnagelvin Area Hospital, Derry, Northern Ireland, UK

${ }^{5}$ School of Sport, Ulster University, Northern Ireland, UK

${ }^{6}$ Applied Sports Technology, Exercise and Medicine Research Centre, Swansea University, Bay Campus, Fabian Way, Swansea, SA1 8EN, UK

*Corresponding Author

Richard Metcalfe

Applied Sports Technology, Exercise and Medicine Research Centre (A-STEM)

Swansea University

Bay Campus

Fabian Way

Swansea

SA1 8EN

UK

Email: r.s.metcalfe@,swansea.ac.uk 


\section{Author Contribution}

RM, NV and PS conceived the study ideas, designed the studies, collected the data, and wrote the initial draft of the manuscript. BF, SF, NB, CM, GM and GD offered critical insight during the study design (study 2), supported data collection (study 2), and provided critique of the initial manuscript draft. All authors approved the final version of the manuscript. RM is the guarantor of this work.

\section{Acknowledgements}

We would like to thank our participants for their time and effort in completing this study, and Lewis Darnborough, Gregor Barr, Patrick Muirhead and Paul Carson for assistance with data collection for study 1, Diane Fernie for her help with study 2, and James Bolam, Catherine Henderson, Ross McGinness, Greg Wallace, Joe Senior, Sean Ayoade and Chris May for assistance in training supervision for study 3 . We would also like to extend a special thank you to our family and friends for their support during our academic endeavours.

\section{Funding Details}

This writing of this manuscript was supported by The Diabetes Research and Wellness Foundation (SCA/OF/12/15). PS was supported by a studentship from Thammasat University. GM was supported by a summer studentship from The Physiological Society. We would also like to acknowledge the financial support of Swansea University, Stirling University and Ulster University.

\section{Competing Interests}

We have no conflict of interest to declare. 


\section{Abstract}

We have previously demonstrated that reduced-exertion high-intensity interval training (REHIT) is a genuinely time-efficient exercise strategy for improving cardiometabolic health. Here, we examined the affective and perceptual responses to REHIT. Eight young men and women (age $21 \pm 1 \mathrm{y}$, BMI $24.9 \pm 2.1 \mathrm{~m} / \mathrm{kg}^{2}, \dot{\mathrm{V}}{ }_{2} \max 39 \pm 10 \mathrm{ml} / \mathrm{kg} / \mathrm{min}$ ) and $11 \mathrm{men}$ with type 2 diabetes (T2D; age $52 \pm 6$ y, BMI $29.7 \pm 3.1 \mathrm{~m} / \mathrm{kg}^{2}, \dot{\mathrm{V}} \mathrm{O}_{2} \mathrm{max} 29 \pm 5 \mathrm{ml} / \mathrm{kg} / \mathrm{min}$ ) took part in three-arm crossover trials with RPE and affective valence measured during, and enjoyment and exercise preferences measured following either: 1) REHIT (2x20-s sprints in a 10-min exercise session), 2) HIIT (10x1-min efforts) and 3) 30 min MICT. Furthermore, 19 young men and women (age $25 \pm 6 \mathrm{y}$, BMI $24 \pm 4 \mathrm{~m} / \mathrm{kg}^{2}, \dot{\mathrm{VO}} 2 \max 34 \pm 8 \mathrm{ml} / \mathrm{kg} / \mathrm{min}$ ) completed a 6-week REHIT intervention with affective valence during an acute REHIT session measured before and after training. Affect decreases (briefly) during REHIT, but recovers rapidly, and the decline is not significantly different when compared to MICT or HIIT in either healthy participants or T2D patients. Young sedentary participants reported similar levels of enjoyment for REHIT, MICT and HIIT, but 7 out of 8 had a preference for REHIT. Conversely, T2D patients tended to report lower levels of enjoyment with REHIT compared with MICT. The decrease in affective valence observed during an acute REHIT session was significantly attenuated following training. We conclude that affective and perceptual responses to REHIT are no more negative compared to those associated with MICT or HIIT, refuting claims that supramaximal sprint interval training protocols are associated with inherent negative responses.

Key words: Exercise. Interval Training. Affect. Perceived Exertion. 


\section{Abbreviations}

BMI, Body Mass Index. $\mathrm{CO}_{2}$, carbon dioxide. HRmax, maximal heart rate. HIIT, high intensity interval training. IPAQ, International Physical Activity Questionnaire. MICT, moderate intensity continuous exercise. $\mathrm{O}_{2}$, oxygen. PACES, Physical Activity Enjoyment Scale. REHIT, reduced-exertion high intensity interval training. RPE, ratings of perceived exertion. SIT, sprint interval training. T2D, type 2 diabetes. $\dot{\mathrm{V}} \mathrm{O}_{2} \mathrm{max}$, maximal aerobic capacity. $\dot{\mathrm{V}} \mathrm{O}_{2}$, oxygen uptake. Wmax, peak power output. 


\section{Introduction}

Regular exercise can be considered a prerequisite for good long-term health, whilst physical inactivity is strongly associated with an increased risk of chronic cardiometabolic disease and premature death (Booth, Roberts, \& Laye, 2012; Booth, Roberts, Thyfault, Ruegsegger, \& Toedebusch, 2017). However, despite more than two decades of promoting specific physical activity guidelines which have been based (predominantly) on performing 30 min of moderate intensity continuous exercise (MICT) on most days of the week (Blair, LaMonte, \& Nichaman, 2004; Garber et al., 2011), a large proportion of the general population still do not participate in adequate levels of clinically meaningful exercise on a regular basis (Allender et al., 2008; Colley et al., 2011; Hallal et al., 2012).

There are numerous and complex interacting barriers which contribute to the poor uptake of and adherence to exercise in the general population, but one common reported barrier is a perceived lack of time (Korkiakangas, Alahuhta, \& Laitinen, 2009; Reichert, Barros, Domingues, \& Hallal, 2007). To address this barrier, much research has focussed on (sub)maximal high-intensity interval training (HIIT) and supramaximal sprint interval training (SIT), as potential alternatives to MICT for modifying cardiometabolic disease risk factors (Vollaard \& Metcalfe, 2017). There is now clear evidence that both HIIT and SIT can produce similar adaptations compared to MICT with a substantially lower exercise volume (Burgomaster, Heigenhauser, \& Gibala, 2006; Burgomaster, Hughes, Heigenhauser, Bradwell, \& Gibala, 2005; Ciolac et al., 2010; Gibala et al., 2006; MacInnis et al., 2017; Tjønna et al., 2008). However, the true time-efficiency of the most commonly studied HIIT ( $\sim 25$ min per session; (Gillen, Percival, Ludzki, Tarnopolsky, \& Gibala, 2013; Little et al., 2011)) and SIT protocols ( $\sim 30$ min per session; (Burgomaster et al., 2006; Burgomaster et al., 2005; Gibala et 
al., 2006)) can be questioned. Furthermore, there is currently vigorous debate about whether either would be appropriate exercise strategies for recommendation to the general population. Both are assumed to be experienced as 'unpleasant' due to high perceived exertion and negative affective responses (Biddle \& Batterham, 2015; Hardcastle, Ray, Beale, \& Hagger, 2014); i.e. HIIT and SIT may be effective, but they are predicted not to be acceptable. Specifically, critics of HIIT/SIT cite studies which demonstrate that affective valence decreases to a greater extent when inactive people participate in vigorous compared with moderate intensity exercise (Ekkekakis, Hall, \& Petruzzello, 2005, 2008; Ekkekakis, Lind, \& Vazou, 2010; Ekkekakis, Parfitt, \& Petruzzello, 2011). Thus, it is argued that inactive individuals would find the required high exercise intensities with HIIT/SIT less enjoyable and less preferable compared with MICT (Biddle \& Batterham, 2015; Hardcastle et al., 2014). As exercise-induced negative affect has been associated with future avoidance of exercise and physical activity (Brand \& Ekkekakis, 2018; Rhodes \& Kates, 2015; Williams et al, 2008), in theory this would lead to poor longterm adherence to HIIT and SIT interventions. However, firstly, it is important to highlight that the cited studies on changes in affect with exercise compared continuous vigorous and moderate intensity exercise (Ekkekakis et al., 2008; Ekkekakis et al., 2010; Ekkekakis et al., 2011) and do not necessarily translate to vigorous intensity exercise when performed in short intervals interspersed with adequate recovery periods (Jung, Bourne, \& Little, 2014). Secondly, the influence of exercise duration needs to be taken into account. A clear finding from studies on the acute responses to both MICT and HIIT/SIT protocols is that perceived exertion increases as a function of time (MICT; (Kearon, Summers, Jones, Campbell, \& Killian, 1991)), and of the number and duration of the high-intensity efforts (Frazão et al., 2016; Kilpatrick et al., 2015; Martinez, Kilpatrick, Salomon, Jung, \& Little, 2015; Townsend et al., 2017). Similarly, affect decreases progressively with repeated high intensity efforts in HIIT/SIT protocols (Dekker \& Ekkekakis, 2017; Frazão et al., 2016; Kilpatrick et al., 2015; Martinez et 
al., 2015; Niven, Thow, Holroyd, Turner, \& Phillips, 2018; Stork, Gibala, \& Martin Ginis, 2018; Townsend et al., 2017), while it may also decrease over time with MICT, depending on the intensity (Dekker \& Ekkekakis, 2017; Ekkekakis et al., 2010). Thus, it follows that as long as HIIT/SIT protocols can limit the number and duration of high intensity efforts, affective valence may decrease to a similar or lower extent compared to MICT, and could therefore be perceived more favourably by inactive individuals. Indeed, several, although not all (Dekker \& Ekkekakis, 2017), studies have reported that participants find HIIT/SIT similarly (Green et al., 2017; Jung et al., 2014; Vella, Taylor, \& Drummer, 2017) or more (Malik, Williams, Bond, Weston, \& Barker, 2017; Thum, Parsons, Whittle, \& Astorino, 2017) enjoyable when compared with MICT.

In a series of studies we have demonstrated that reducing the number of sprint repetitions (from 6-7 to 2) and the sprint duration (from 30 to 20 seconds) in Gibala's 'classic' SIT protocol (Burgomaster et al., 2006; Burgomaster et al., 2005; Gibala et al., 2006) does not attenuate the improvements in important markers of cardiometabolic health, including maximal aerobic capacity (Metcalfe, Tardif, Thompson, \& Vollaard, 2016; Nalçakan et al., 2018; Vollaard, Metcalfe, \& Williams, 2017), insulin sensitivity and glycaemic control (Metcalfe, Babraj, Fawkner, \& Vollaard, 2012; Metcalfe et al., 2018), and blood pressure (Ruffino et al., 2017). The resulting SIT protocol (termed 'reduced-exertion high-intensity interval training' (REHIT)), is genuinely time-efficient, involving 2 x 20 -s sprints in a 10-min exercise session. Our findings are important because they accomplish the overarching aim of HIIT/SIT protocols to provide a time-efficient exercise protocol, while at the same time reducing the likelihood of negative affect resulting in reduced exercise enjoyment. Indeed, a modified REHIT protocol was recently shown to be as enjoyable and preferable as MICT among inactive individuals (Stork et al., 2018). Furthermore, we have recently demonstrated that REHIT is not associated with an increase in negative affect in the immediate post-exercise period, and in contrast 
increases (with small effect sizes) positive affect and overall mood state (Nalçakan et al., 2018). Nonetheless, prior to efforts to implement REHIT as a 'real-life' practical exercise intervention for inactive healthy and patient populations, there is a need for a better understanding of the acute and chronic perceptual, affective and enjoyment responses to REHIT.

Thus, in the present series of independent studies we first aimed to explore the acute perceptual, affective and enjoyment responses to REHIT in young sedentary but otherwise healthy participants, and compared these to current MICT recommendations (Garber et al., 2011) and to one of the most commonly studied low volume HIIT protocols (Little et al., 2011). We subsequently explored the acute responses to REHIT in a cohort of sedentary middle-aged men with type 2 diabetes, and compared these to MICT and low volume HIIT. Finally, we explored whether the acute perceptual and affective responses to REHIT are modified by training. 


\section{Methods}

\section{Study 1: Acute Responses in Healthy Sedentary Participants}

\section{Participants:}

Eight healthy young sedentary or recreationally active men and women were recruited to participate in study 1 (7 men and 1 women: mean and SD: age $21 \pm 1 \mathrm{y}$, BMI $24.9 \pm 2.1 \mathrm{~m} / \mathrm{kg}^{2}$, $\left.\dot{\mathrm{V}} \mathrm{O}_{2} \max 39 \pm 10 \mathrm{ml} / \mathrm{kg} / \mathrm{min}\right)$. Participants with prior diagnosis of chronic cardiometabolic disease, absolute contraindications to exercise determined using the PAR-Q (Thomas, Reading, \& Shephard, 1992), classified as highly physically activity on the IPAQ (Craig et al., 2003), currently taking mood-altering medications (e.g. anti-depressants), or with clinically significant hypertension ( $>140 / 90 \mathrm{~mm} \mathrm{Hg}$ ), were excluded from participation. All participants gave their written informed consent to participate in the study, which was approved by the Stirling University Ethics Committee (NICR (17/18) Paper No. 17).

\section{Study Design and Experimental Procedures:}

Participants attended the laboratory on 4 occasions prior to the main experiment. During the first visit, participants' maximal aerobic capacity ( $\left.\dot{\mathrm{V}} \mathrm{O}_{2} \mathrm{max}\right)$, peak power output (Wmax) and maximal heart rate (HRmax) were determined during an incremental cycling test (1 W increase every $3 \mathrm{~s}$ ) to exhaustion on an electronically braked cycle ergometer (Excalibur Sport, Lode, Groningen, the Netherlands), with breath-by-breath measurement of oxygen uptake using an online gas analyser (Oxycon Pro, Jaeger, Wurzburg, Germany). After this, participants completed three separate sessions, separated by at least 2 days, where they were familiarised with the three exercise conditions. In these sessions, participants performed progressively longer durations of moderate-intensity continuous exercise at $40 \%$ of $\mathrm{Wmax}(5$, then 10 , and then 15 minutes in session 1, 2 and 3, respectively), progressively more high intensity efforts 
in the high-intensity interval training protocol (1, then 3 , and then 5 efforts in session 1, 2 and 3, respectively), and progressively longer durations of the 'all-out' sprints used in the REHIT protocol (10, then 15, and then 20 seconds in session 1, 2 and 3, respectively).

At least 1 week after completion of the familiarization sessions, participants commenced a randomized crossover study comparing three exercise conditions: MICT (30 minutes of cycling at $40 \%$ of Wmax (91 \pm 20 watts), HIIT (10 x 1-min cycle efforts at $100 \%$ of Wmax $(229 \pm 49$ watts) within a 22-min exercise session (Gillen et al., 2013; Little et al., 2011)), and REHIT (2 x 20-s 'all-out' cycle sprints (against a resistance of $7.5 \%$ body mass) within a 10 -min exercise session (Metcalfe, Fawkner, \& Vollaard, 2016; Metcalfe et al., 2012; Metcalfe et al., 2015; Metcalfe et al., 2016; Nalçakan et al., 2018)). Affective valence (eleven-point feeling scale (Hardy \& Rejeski, 1989) and ratings of perceived exertion (RPE; 15-point Borg scale (Borg, 1982)) were assessed before exercise, every 2 min during exercise, and then 10 and 30 min post-exercise. The sprints during REHIT were performed between 1:40-2:00 and 5:40-6:00 to ensure that the most intense exercise was captured within the RPE and affect measurements (measurements were taken immediately on completion of the sprints). Furthermore, exercise enjoyment (Physical Activity Enjoyment Scale (PACES); (Kendzierski \& DeCarlo, 1991)) was assessed 30 min post-exercise. The PACES scale was modified by removing the item 'current absorption in the activity' and adding 2 questions on current and expected future enjoyment of the exercise, as previously proposed by Jung et al (Jung et al., 2014). Heart rate was measured during exercise by telemetry (Polar RS400, Kempele, Finland). Preference of exercise protocol was determined using the procedure proposed by Townsend et al (2017). Specifically, participants were told that they would complete a fourth experimental condition where, upon arrival for the exercise session, they would be asked to choose which of the 3 exercise protocols they would prefer to perform. Following their selection, participants were informed that they did not actually need to perform the exercise, but their choice was taken as an indicator of 
preference. Seven participants completed all exercise sessions in full, but one participant experienced nausea during HIIT and hence the exercise session was terminated early. As such, RPE and affective responses over time are presented for $n=7$.

\section{Study 2: Acute Responses in Sedentary Middle-Aged Participants with Type 2 Diabetes}

\section{Participants:}

These data were secondary outcomes from a study investigating the effects of MICT, HIIT and REHIT on glycaemic control (ClinicalTrials.gov registration: NCT03082859). The experimental protocol was approved by the Office for Research Ethics in Northern Ireland (RECA ref: 16/NI/0115). Eleven men, diagnosed with type 2 diabetes by a clinician at least 3 months previously, completed the full experimental procedures (mean and SD: age $52 \pm 6 \mathrm{y}$, BMI $29.7 \pm 3.1 \mathrm{~m} / \mathrm{kg}^{2}, \dot{\mathrm{V}}{ }_{2} \max 29 \pm 5 \mathrm{ml} / \mathrm{kg} / \mathrm{min}$, fasting glucose $8.1 \pm 1.2 \mathrm{mmol} / \mathrm{L}, \mathrm{HbA} 1 \mathrm{c}$ $7.0 \pm 0.8 \%$ ). Exclusion criteria included exogenous insulin therapy, currently taking more than

2 glucose-lowering medications, BMI $\geq 40 \mathrm{~kg} / \mathrm{m}^{2}$, classification as highly active on the International Physical Activity Questionnaire (IPAQ) (Craig et al., 2003), and any contraindications to exercise, including any history of cardiovascular or cerebrovascular disease, impaired renal or liver function, and hypertension not well controlled by standard medication. All participants were informed about the study, both verbally and in writing, before providing their written consent to participate. Eligible participants completed a 12-lead exercise stress test on a cycle ergometer (Lode Corival; Lode, Groningen, Netherlands) and received clearance for vigorous intensity exercise from a clinical cardiac physiologist.

\section{Study Design and Experimental Procedures}

Following health screening, participants completed a maximal incremental cycling test to volitional exhaustion to determine maximal aerobic capacity $\left(\dot{\mathrm{V}}_{2} \mathrm{max}\right)$, peak power output 
(Wmax) and maximal heart rate (HRmax). Following a 5 min warm up at $50 \mathrm{~W}$, the intensity was increased by $15 \mathrm{~W} / \mathrm{min}$ until cadence could not be maintained at $\geq 50 \mathrm{rpm}$ (Lode Corival, Groningen, The Netherlands). $\mathrm{V}_{2} \max$ was determined as the highest 10-breath rolling average of $\dot{\mathrm{VO}}_{2}$ measured using an online gas analysis system (Cosmed Quark; CPET, Rome, Italy). Participants also completed two familiarisation sessions, on separate days, each lasting approximately $20 \mathrm{~min}$, to introduce participants to the technique and effort required to perform the all-out cycling sprints used during REHIT. Participants completed the REHIT exercise session in full on both occasions, with 10 -s sprints in the first and 20 -s sprints in the second session.

Participants then completed three exercise trials in a randomised order: 1) HIIT (10 x 1-min at $85 \%$ Wmax, 23 min total time commitment); 2) REHIT ( $2 \times 20$-s sprints against a resistance of 5\% body mass, $10 \mathrm{~min}$ total time commitment); and 3) MICT (30 min at 50\% Wmax). Participants also completed a fourth no-exercise control trial as part of the experiment, but no data relevant to this manuscript were collected. Each trial was separated by at least 5 days and participants avoided strenuous or tiring physical activity for at least 2 days before the trial. Participants performed the exercise session at the same time in the morning (between 08:00

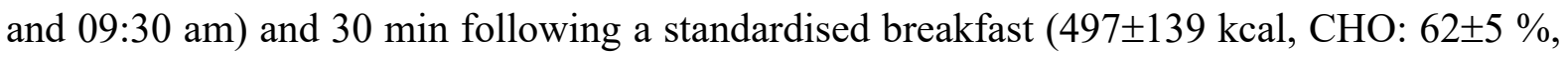
fat: $21 \pm 5 \%$, protein: $17 \pm 6 \%$ ).

Affective valence (eleven-point feeling scale (Hardy \& Rejeski, 1989) and RPE (15-point Borg scale (Borg, 1982)) were assessed prior to exercise, every 3 min during MICT and every 1 min during both REHIT and HIIT, and then $10 \mathrm{~min}, 20 \mathrm{~min}$ and $30 \mathrm{~min}$ following cessation of exercise. The sprints during REHIT were performed at 2:40-3:00 and 6:40-7:00 so that the most intense exercise was captured (measurements were taken immediately on completion of the sprints). Furthermore, exercise enjoyment (unmodified PACES; (Kendzierski \& DeCarlo, 
1991)) was assessed immediately post-exercise with participants resting comfortably in a chair. Heart rate was measured by telemetry (Polar RS400, Kempele, Finland) throughout each exercise session.

\section{Study 3: Training-Induced Changes in the Responses in Healthy Sedentary Participants}

\section{Participants}

These data were collected as part of an ongoing study at Swansea University and Stirling University of the effects of training frequency on adaptations in maximal aerobic capacity with REHIT. Nineteen healthy young sedentary or recreationally active men $(n=10)$ and women $(\mathrm{n}=9)$ took part in this study (mean and SD: age $25 \pm 6 \mathrm{y}, \mathrm{BMI} 24 \pm 4 \mathrm{~m} / \mathrm{kg}^{2}, \dot{\mathrm{VO}}{ }_{2} \mathrm{max} 34 \pm 8$ $\mathrm{ml} / \mathrm{kg} / \mathrm{min}$ ). Participants with any prior diagnosis of chronic cardiometabolic disease, any other absolute contraindications to exercise determined using the PAR-Q (Thomas et al., 1992), classified as highly physically activity on the IPAQ (Craig et al., 2003), or with clinically significant hypertension (>140/90 mmHg), were excluded from participation. All participants gave their written informed consent to participate in the study which was approved by the local University ethics committees (Stirling Approval Reference: NICR (17/18) Paper No. 16; Swansea Approval Reference: 2016-111).

\section{Study Design and Experimental Procedures}

All participants performed an incremental cycling test ( $1 \mathrm{~W}$ every $3 \mathrm{~s})$ to volitional exhaustion on an electronically braked cycle ergometer (Lode Excalibur Sport, Groningen, The Netherlands). Expired $\mathrm{O}_{2}$ and $\mathrm{CO}_{2}$ were determined continuously throughout the test using an online gas analyser, and $\dot{\mathrm{V}}_{2} \max$ was determined as the highest value for a 15-breath rolling

average of $\mathrm{VO}_{2}$. $\dot{\mathrm{V}} \mathrm{O}_{2}$ max was accepted if $\mathrm{RER}>1.10$ and heart rate was within 10 beats of the age-predicted maximum (i.e. 220-age). 
At least 2 days following the $\dot{\mathrm{V}} \mathrm{O}_{2}$ max test participants started a 6-week REHIT intervention consisting of 2, 3 or 4 training sessions per week. Training sessions consisted of 10 minutes of unloaded pedalling, with two 'all-out' cycle sprints against a resistance of $7.5 \%$ of the participant's body mass. The duration of the sprints was increased from $10 \mathrm{~s}$ in week 1 , to 15 $\mathrm{s}$ in week 2 , and then to $20 \mathrm{~s}$ in the final 4 weeks of training. Affective valence (eleven-point feeling scale (Hardy \& Rejeski, 1989)) and RPE (15-point Borg scale (Borg, 1982)) were assessed every min during exercise in the first 20-s training session (the first session of week 3) and then during the final training session (the final session of week 6). Sprints were completed between 1:40-2:00 min and 5:40-6:00 min to ensure that RPE and affect were captured immediately following the most intense exercise.

\section{Statistical Analysis}

All statistical analysis was performed in IBM SPSS (version 22). Data are presented as mean \pm SD unless otherwise stated. Significance was accepted at $p<0.05$. Where relevant, Cohens $d$ was calculated as a measure of effect size (mean difference/pooled variance) with the following thresholds: $>0.2$ small, $>0.6$ moderate and $>1.2$ large (Hopkins, Marshall, Batterham, \& Hanin, 2009).

\section{Study 1 and 2}

Enjoyment and summary variables for RPE (peak) and affective valence (lowest, end and change (Stork, Banfield, Gibala, \& Martin Ginis, 2017)) were analysed by a one-factor (condition) repeated measures ANOVA regardless of minor deviations from a normal distribution and with the Greenhouse Geisser correction applied in cases of violated sphericity (Maxwell \& Delaney, 2004). In the case of significant main effects, then post-hoc comparisons were performed using Fishers LSD (i.e. uncorrected paired t-tests) since there is no inflation 
of type 1 error rates following a significant main effect when only three comparisons are being made (Howell, 1997).

\section{Study 3}

The effect of training on the affective and perceptual response to acute exercise was examined with a two-way (exercise time $\times$ training) repeated measures ANOVA regardless of minor deviations from a normal distribution and with the Greenhouse Geisser correction applied in cases of violated sphericity (Maxwell \& Delaney, 2004). If appropriate, pre-planned contrasts (pre $v s$ post at each exercise time point) were compared using Fishers LSD (i.e. uncorrected paired t-tests) post-hoc tests. Comparisons of RPE (peak) and affective valence (lowest and change) summary variables were made using paired sample t-tests. 


\section{Results}

\section{Acute Responses in Young Sedentary Participants}

We first explored the RPE (Figure 1A) and affective valence (Figure 1B and 1C) responses to MICT, HIIT and REHIT in healthy sedentary participants. Both mean and peak heart rate were lowest during MICT (mean $65 \pm 4 \%$ HRmax, peak $72 \pm 5 \%$ HRmax), higher during REHIT (mean $71 \pm 6 \%$ HRmax, peak $90 \pm 5 \%$ HRmax; $p=0.042$ and $p<0.001$, respectively $v s$ MICT) and highest during HIIT (mean $83 \pm 6 \%$ HRmax, peak 96 $\pm 5 \%$ HRmax; $p<0.001$ and $p=0.034$, respectively $v s$ MICT; $p=0.007$ and $p<0.001$ respectively $v s$ REHIT). There was a significant main effect of exercise condition for peak RPE $(p=0.002)$. Post-hoc analysis revealed similar peak RPE between HIIT and REHIT $(16.3 \pm 2.4$ and $17.0 \pm 1.9$, respectively, $p=0.622)$ and in both cases this was higher when compared with MICT (11.0 $\pm 2.1, p<0.001 v s$ HIIT and $p=0.003$ vs REHIT, respectively; Figure 1A). There was no significant main effect of exercise condition on the change in affect (MICT: $-2.1 \pm 1.6$ vs HIIT: $-3.9 \pm 1.9$ vs REHIT: $-2.7 \pm 3.0, p=0.375$ ) and only a trend toward a significant main effect for the lowest reported affect (MICT: $0.7 \pm 1.4 v s$ HIIT: $-1.3 \pm 2.8$ vs REHIT: $0.6 \pm 2.4, p=0.080$ ) observed during exercise (Figure $1 \mathrm{~B}$ and $1 \mathrm{C}$ ). However, there were moderate effect sizes favouring MICT when compared with HIIT (lowest: $d=0.85$, change: $d=0.78$ ) and small to moderate effect sizes favouring REHIT when compared with HIIT (lowest: $d=0.79$, change: $d=0.52$ ). Differences between REHIT and MICT were negligible or small (lowest: $d=0.06$, change: $d=0.26$ ). There was also no main effect for end affect (MICT: $0.9 \pm 1.3 v s$ HIIT: $1.7 \pm 2.1 v s$ REHIT: $2.0 \pm 2.2, p=0.301$ ). The effect size for the difference in end affect was moderate favouring REHIT compared with MICT ( $d=0.61)$, small favouring HIIT compared with MICT $(d=0.46)$, and negligible for REHIT compared with HIIT $(d=0.15)$. There were no differences in levels of exercise enjoyment reported between the exercise conditions (MICT: $70 \pm 16$ vs HIIT: $80 \pm 19$ vs REHIT: $87 \pm 22, p=0.177$ for main effect). 
The effect size for differences in enjoyment was moderate favouring REHIT compared with MICT $(d=0.86)$, small favouring REHIT compared with HIIT $(d=0.34)$, and small favouring HIIT compared with MICT $(d=0.52)$. On attendance for the (fictitious) fourth exercise session, 7 out of 8 participants selected to perform REHIT, and the remaining participant selected to perform HIIT. None selected to perform MICT.

\section{Acute Responses in Middle-Aged Participants with Type 2 Diabetes}

Apart from use in prevention of noncommunicable diseases, exercise can also be employed in the treatment of patients with a range of conditions, including type 2 diabetes. Such patient populations may exhibit different acute responses to exercise, and therefore we next explored the RPE (Figure 2A) and affective (Figure 2B and 2C) response in 11 participants with type 2 diabetes. During the exercise work intervals, mean power output was lowest during MICT (97 $\pm 17 \mathrm{~W})$, higher during HIIT $(165 \pm 28 \mathrm{~W}, p<0.001$ vs MICT), and higher still following REHIT (417 $\pm 49 \mathrm{~W}, p<0.001$ vs HIIT and MICT, respectively). Peak exercise heart rate tended to be lower during MICT ( $87 \pm 7 \%$ HRmax) and REHIT (90 $\pm 3 \%$ HRmax) compared with HIIT (94 $\pm 5 \%$ HRmax, $p=0.058$ and $p=0.004$ vs REHIT and MICT, respectively), whilst mean HR was lowest during REHIT (73 $\pm 6 \% \mathrm{HRmax}$ ), higher during MICT (77 $\pm 6 \%$ HRmax, $p=0.021$ $v s$ REHIT), and highest during HIIT ( $80 \pm 6 \%$ HRmax, $p=0.002$ and $p=0.021$ ss REHIT and MICT, respectively). There was a significant main effect of exercise condition for peak RPE $(p<0.001)$. Post-hoc analysis revealed a higher peak RPE during REHIT (17.7 \pm 1.9$)$ compared with both MICT $(14.7 \pm 3, p=0.001)$ and HIIT $(15.5 \pm 2.0, p=0.002$; Figure $2 \mathrm{~A})$, respectively. There were no main effects for the lowest reported affect (MICT: $0.1 \pm 2.4 v s$ HIIT: $-0.3 \pm 2.7 v s$ REHIT: $-1.0 \pm 3.0, p=0.103$ ), for the change in affect observed during exercise (MICT: $-4.0 \pm 2.0$ vs HIIT: $-4.3 \pm 2.7 v s$ REHIT: $-4.9 \pm 2.5, p=0.292$ ), or for affect at the end of exercise (MICT: $0.6 \pm 2.8 v s$ HIIT: $1.6 \pm 2.4 v s$ REHIT: $2.3 \pm 2.8, p=0.076$; Figure $2 \mathrm{~B}$ and $2 \mathrm{C}$ ). Except for a 
moderate effect size favouring REHIT compared with MICT for end exercise affect $(d=0.61)$, the effect sizes for differences between conditions were small or negligible (range: 0.12-0.41). There was a trend toward a main effect of condition on exercise enjoyment (REHIT $83 \pm 16 v s$ HIIT $86 \pm 15$ vs MICT $90 \pm 14, p=0.059)$ which largely appeared to be driven by lower enjoyment scores for REHIT compared with MICT ( $p=0.013, d=0.52)$.

\section{Effect of Training on the Acute RPE and Affective Response to REHIT}

Although the acute perceptual responses to exercise are believed to be informative about the likelihood of individuals taking up and adhering to an exercise routine, there are indications that these responses can change over time during a training intervention (Saanijoki et al., 2018). Thus, we next explored the effect of 6 weeks of training on the acute responses to REHIT. There were main effects of exercise time for RPE $(p<0.001)$ and the affective response $(p<0.001)$ demonstrating the increase in RPE and decrease in affect during an acute bout of REHIT (Figure 3A and 3B). There were no main effects of training on the RPE $(p=0.678)$ and affective response $(p=0.263)$ and there was also no significant training $\mathrm{x}$ time interaction present for RPE ( $p=0.073$; Figure $3 \mathrm{~A})$. However, there was a significant training $\mathrm{x}$ time interaction present for affect ( $p=0.006$ ) suggesting that training attenuated the decrease in affect during acute exercise (Figure 3B). Post-hoc analysis revealed that training resulted in more positive affect immediately $(0.5 \pm 2.8 v s-1.0 \pm 2.4, p=0.012, d=0.57)$ and one-minute following $(1.5 \pm 2.4$ vs $0.6 \pm 2.5, p=0.049, d=0.34)$ the second sprint (Figure 3B). The lowest reported affect during exercise was more positive following training $(0.1 \pm 2.9 v s-1.1 \pm 2.2, p=0.034, d=0.46)$ and there was also a less exaggerated change in affect during exercise $(-2.8 \pm 2.2 v s-4.1 \pm 2.1$, $p=0.005, d=0.58)$. 


\section{Discussion}

The aim of the present study was to explore the acute and chronic perceptual, affective and enjoyment responses to REHIT in different populations and in comparison to MICT and a commonly-studied HIIT protocol. The main findings of the current paper are threefold. Firstly, in agreement with a recent report (Stork et al., 2018), our data show that although affect does (briefly) decrease during REHIT, it recovers rapidly. Importantly, the decline in affect is of a similar magnitude compared with MICT and HIIT in both healthy sedentary participants and participants with type 2 diabetes. Secondly, young sedentary participants report similar levels of enjoyment for REHIT, MICT and HIIT (with a moderate effect size favouring REHIT $v s$ MICT), and the majority of our participants (7 out of 8) recorded a preference for REHIT. Conversely, middle-aged participants with type 2 diabetes tended to report lower levels of enjoyment with REHIT compared with MICT. Finally, we show that the decrease in affective valence observed during an acute REHIT session is attenuated following the initial weeks of a training intervention. Taken together, these findings are important because, contrary to current opinion (Hardcastle et al., 2014), they demonstrate that SIT protocols incorporating supramaximal intensity efforts are not necessarily perceived negatively when the number and duration of sprints is kept low.

Despite the extreme divergence in exercise intensities, there were no significant differences in the lowest reported affect or the change in affect between REHIT, HIIT and MICT. Notably, in the healthy sedentary cohort there was a moderate effect size favouring REHIT and MICT compared with HIIT for both affect summary variables, suggesting that HIIT resulted in more negative affective valence. These findings oppose the argument of detractors of HIIT/SIT that performing repeated high intensity efforts/sprints will be a universally unpleasant experience due to negative affective responses, whilst performing MICT will be a universally more 
pleasant experience (Biddle \& Batterham, 2015; Dekker \& Ekkekakis, 2017; Hardcastle et al., 2014). We demonstrate that if the number and duration of sprint repetitions is kept low, SIT protocols are no less pleasant than MICT. We propose that a key consideration (which is rarely acknowledged) is the interaction between the absolute decrease in affective valence and the overall 'exposure' to low levels of affective valence dependent on exercise duration. Indeed, the duration of the exercise efforts with REHIT is (intentionally) substantially reduced compared with MICT and hence the overall exposure to lower levels of affective valence with REHIT is brief. Whilst the absolute decrease in affect is clearly an important consideration (Hargreaves \& Stych, 2013; Williams et al, 2008), the overall exposure to high RPE and lower affective valance will also arguably be important in informing a person's overall perceptions of an exercise experience (Brand \& Ekkekakis, 2018). It is reasonable to suggest that a few seconds at a high RPE or a low affective valence (e.g. with REHIT) would not be considered more unpleasant than 30 minutes at a slightly lower RPE or slightly higher affective valence (e.g. with MICT). Regardless, the present study shows similar absolute decreases in affective valence, but clearly far less exposure to lower levels of affective valence, with REHIT compared with both MICT and HIIT. When combined with the overall time-efficiency of REHIT, this presumably (at least partly) explains the similar (possibly higher based on effect size) levels of enjoyment, and greater preferences for REHIT, compared with MICT and HIIT in our healthy sedentary cohort.

Our study extends the findings by Stork et al (2018) who recently studied the perceptual responses to a modified REHIT protocol in a similar cohort of participants. In this study, and other work by this group (Gillen et al., 2016; Gillen et al., 2014), our original REHIT protocol was modified to include a third 20-s sprint, presumably based on the persistent but incorrect (Vollaard et al., 2017) assumption that a larger SIT exercise volume will be associated with more pronounced adaptations. They reported that affective valence decreased progressively 
(by $\sim 1$ point on the feeling scale) with each 'all-out' 20 -s sprint and, in contrast to our findings, that REHIT was associated with more negative affective valence compared with MICT (Stork et al., 2018). The more negative affective response with REHIT reported in their study can be explained by: 1) the incorporation of the third sprint (which is unnecessary for achieving improvements in cardiometabolic risk factors (Metcalfe et al., 2012; Metcalfe et al., 2016; Nalçakan et al., 2018; Vollaard et al., 2017)), and 2) the lack of sufficient familiarisation with REHIT prior to the main experiment. In our REHIT training studies (Metcalfe et al., 2012; Metcalfe et al., 2016; Nalçakan et al., 2018), we have observed that progressively increasing sprint duration from $10 \mathrm{~s}$ to $20 \mathrm{~s}$ over the first few sessions completely prevents the feelings of nausea which were reported in early studies of 'classic' SIT. Therefore, in the present study we included three familiarisation sessions with participants performed increasing duration of allout sprints during each.

The decrease in affect observed during an acute REHIT session was significantly attenuated following a period of exercise training. This is in agreement with a recent report from Saanijoki et al (Saanijoki et al., 2018) who reported that the acute affective response to 'classic' SIT (4 x 30-second sprints) was improved following just six SIT sessions in sedentary and insulin resistant individuals. From a practical perspective, this provides further support for the 'lead in' period that we include in the REHIT protocol (i.e. gradually building up the sprint duration from 10 to 20 seconds over the initial training weeks), as this may improve affective responses during the initial training sessions prior to starting the more demanding 20 -s sprints.

We observed no differences in affective valence summary variables between exercise conditions in our sample of sedentary overweight men with type 2 diabetes, but in contrast to our young healthy sedentary cohort, participants with type 2 diabetes tended to report lower levels of enjoyment with REHIT compared with MICT. The perceptual responses to exercise 
are known to be modified by fitness and physical activity status (Frazão et al., 2016) and it is possible that this modifying effect is particularly relevant during exercise requiring higher intensities. Cardiorespiratory fitness was considerably poorer in our type 2 diabetic cohort ( $29 \pm 5 \mathrm{vs} 39 \pm 10 \mathrm{ml} / \mathrm{kg} / \mathrm{min}$ ) compared with our young sedentary cohort and this difference in fitness may explain the divergent findings for exercise enjoyment. However, this finding is somewhat at odds with our previous finding that, following a cross-over intervention study where patients with type 2 diabetes completed both 8 weeks of REHIT and 8 weeks of MICT, twelve out of sixteen participants indicated a preference for REHIT as their intervention of choice (Ruffino et al., 2017). This may potentially be explained by our present finding that the affective responses to REHIT significantly improve during a training intervention. Indeed, enjoyment has been shown to increase progressively during 6 weeks of HIIT but remain constant during 6 weeks of MICT (Heisz, Tejada, Paolucci, \& Muir, 2016). Alternatively, it is possible that shorter exposure duration (40 s for REHIT vs 30 min for MICT) may result in a preference for REHIT despite it being perceived as less enjoyable. Finally, it is also possible that other psychological constructs, not measured in studies 2 and 3, may also have influenced exercise enjoyment. Self-efficacy, or one's beliefs that they can engage in a behaviour, is known to influence enjoyment of higher-intensity exercise activity (Hu, Motl, McAuley, \& Konopack, 2007). Exercise self-efficacy is often low in older patient populations (Bay, Sandberg, Thilén, Wadell, \& Johansson, 2018), possibly influencing the reduced ratings of enjoyment for participants in study 2. Repeated, successful, attempts at a task (i.e. mastery experiences) increase self-efficacy (McAuley \& Blissmer, 2000), however, potentially explaining the increased enjoyment ratings for HIIT in longer-term training interventions (59).

There are a number of strengths and limitations of the current study which should be discussed. Firstly, previous studies comparing perceptual responses to HIIT and MICT have often matched exercise duration or volume (Dekker \& Ekkekakis, 2017), but the need for this is 
unclear for two reasons. Firstly, it undermines the fundamental aim of HIIT to provide a timeefficient alternative to MICT, and, secondly, it is not justified based on the potential adaptive mechanisms (Vollaard \& Metcalfe, 2017). Our previous work on SIT has clearly demonstrated that duration and volume do not relate to magnitude of adaptation for supramaximal exercise (Vollaard et al., 2017). To our mind, a more valid approach, and the one we took in the present studies, is to compare different exercise protocols which are known to be efficacious, no matter how divergent in duration or training volume. This is a strength of the current study. In terms of limitations, firstly we recognise that the relatively small sample size may increase the likelihood of a type 1 error. However, this concern can be somewhat assuaged by the fact that there were no numerical differences in the means and negligible effect sizes for affective valence summary variables between REHIT and the other exercise conditions (i.e. one of our primary comparisons of interest). Secondly, the data from study 2 were secondary outcomes from a trial on glycaemic control and we were only able to include familiarisation sessions for REHIT in this study. The lack of familiarisation for the HIIT and MICT sessions in study 2 may have impacted upon the comparisons of affective responses and results should be interpreted in that context. Finally, during REHIT, we measured RPE and affect on immediate completion of each sprint (rather than within task) because we felt it would be challenging for participants to focus on the scales during an all-out sprint effort. It is possible that a feeling of accomplishment immediately following the sprint could lead to an altered perceptual response, and this possibility should be investigated in future studies on the affective responses to SIT.

To summarise, we show that REHIT is associated with a markedly different pattern of affective response over time compared with both MICT and HIIT. However, we found no evidence that REHIT results in a more negative affective response and is perceived to be similarly enjoyable in young healthy but sedentary participants. In contrast, middle-aged participants with type 2 diabetes tended to report lower levels of enjoyment with REHIT compared with MICT. Finally, 
we also show that the acute affective response to REHIT improves during training. We conclude that SIT protocols incorporating supramaximal intensity efforts are not necessarily perceived negatively when the number and duration of sprints is kept low. Our findings lend further support to our contention that research on the health benefits of SIT and HIIT should focus on protocols with fewer and shorter high-intensity efforts (Vollaard \& Metcalfe, 2017).

\section{Reference List}

Allender, S., Scarborough, P., Peto, V., Rayner, M., Leal, J., Luengo-Fernandez, R., \& Gray, A. (2008). European Cardiovascular Disease Statistics. In. European Heart Network, Brussels, England.

Bay, A., Sandberg, C., Thilén, U., Wadell, K., \& Johansson, B. (2018). Exercise self-efficacy in adults with congenital heart disease. Int $J$ Cardiol Heart Vasc, 18, 7-11. doi:10.1016/j.ijcha.2017.12.002

Biddle, S. J., \& Batterham, A. M. (2015). High-intensity interval exercise training for public health: a big HIT or shall we HIT it on the head? Int J Behav Nutr Phys Act, 12, 95. doi:10.1186/s12966-015-0254-9

Blair, S. N., LaMonte, M. J., \& Nichaman, M. Z. (2004). The evolution of physical activity recommendations: how much is enough? Am J Clin Nutr, 79(5), 913S-920S.

Booth, F. W., Roberts, C. K., \& Laye, M. J. (2012). Lack of exercise is a major cause of chronic diseases. Compr Physiol, 2(2), 1143-1211. doi:10.1002/cphy.c110025

Booth, F. W., Roberts, C. K., Thyfault, J. P., Ruegsegger, G. N., \& Toedebusch, R. G. (2017). Role of Inactivity in Chronic Diseases: Evolutionary Insight and Pathophysiological Mechanisms. Physiol Rev, 97(4), 1351-1402. doi:10.1152/physrev.00019.2016

Borg, G. A. (1982). Psychophysical bases of perceived exertion. Med Sci Sports Exerc, 14(5), 377-381.

Brand, R., \& Ekkekakis, P. (2018). Affective-Reflective Theory of Physical Inactivity and Exercise: Foundations and Preliminary Evidence. German Journal of Exercise and Sport Research, 48(1), 48-58. doi:10.1007/s12662-017-0477-9

Burgomaster, K. A., Heigenhauser, G. J., \& Gibala, M. J. (2006). Effect of short-term sprint interval training on human skeletal muscle carbohydrate metabolism during exercise and time-trial performance. J Appl Physiol, 100(6), 2041-2047. doi:01220.2005 [pii]10.1152/japplphysiol.01220.2005

Burgomaster, K. A., Hughes, S. C., Heigenhauser, G. J., Bradwell, S. N., \& Gibala, M. J. (2005). Six sessions of sprint interval training increases muscle oxidative potential and 
cycle endurance capacity in humans. J Appl Physiol, 98(6), 1985-1990. doi:01095.2004 [pii]10.1152/japplphysiol.01095.2004

Ciolac, E. G., Bocchi, E. A., Bortolotto, L. A., Carvalho, V. O., Greve, J. M., \& Guimarães, G. V. (2010). Effects of high-intensity aerobic interval training vs. moderate exercise on hemodynamic, metabolic and neuro-humoral abnormalities of young normotensive women at high familial risk for hypertension. Hypertens Res, 33(8), 836-843. doi:10.1038/hr.2010.72

Colley, R. C., Garriguet, D., Janssen, I., Craig, C. L., Clarke, J., \& Tremblay, M. S. (2011). Physical activity of Canadian adults: accelerometer results from the 2007 to 2009 Canadian Health Measures Survey. Health Rep, 22(1), 7-14.

Craig, C. L., Marshall, A. L., Sjostrom, M., Bauman, A. E., Booth, M. L., Ainsworth, B. E., . . . Oja, P. (2003). International physical activity questionnaire: 12-country reliability and validity. Med Sci Sports Exerc, 35(8), 1381-1395. doi:10.1249/01.MSS.0000078924.61453.FB

Dekker, E., \& Ekkekakis, P. (2017). More efficient, perhaps, but at what price? Pleasure and enjoyment responses to high-intensity interval exercise in low-active women with obesity. Psychology of Sport and Exercise, 28, 1-10. doi:10.1016/j.psychsport.2016.09.005

Ekkekakis, P., Hall, E. E., \& Petruzzello, S. J. (2005). Variation and homogeneity in affective responses to physical activity of varying intensities: an alternative perspective on doseresponse based on evolutionary considerations. J Sports Sci, 23(5), 477-500. doi:10.1080/02640410400021492

Ekkekakis, P., Hall, E. E., \& Petruzzello, S. J. (2008). The relationship between exercise intensity and affective responses demystified: to crack the 40-year-old nut, replace the 40-year-old nutcracker! Ann Behav Med, 35(2), 136-149. doi:10.1007/s12160-0089025-z

Ekkekakis, P., Lind, E., \& Vazou, S. (2010). Affective responses to increasing levels of exercise intensity in normal-weight, overweight, and obese middle-aged women. Obesity (Silver Spring), 18(1), 79-85. doi:10.1038/oby.2009.204

Ekkekakis, P., Parfitt, G., \& Petruzzello, S. J. (2011). The pleasure and displeasure people feel when they exercise at different intensities: decennial update and progress towards a tripartite rationale for exercise intensity prescription. Sports Med, 41(8), 641-671. doi:10.2165/11590680-000000000-00000

Frazão, D. T., de Farias Junior, L. F., Dantas, T. C., Krinski, K., Elsangedy, H. M., Prestes, J., . . . Costa, E. C. (2016). Feeling of Pleasure to High-Intensity Interval Exercise Is Dependent of the Number of Work Bouts and Physical Activity Status. PLoS One, 11(3), e0152752. doi:10.1371/journal.pone.0152752 
Garber, C. E., Blissmer, B., Deschenes, M. R., Franklin, B. A., Lamonte, M. J., Lee, I. M., .. . Medicine, A. C. o. S. (2011). American College of Sports Medicine position stand. Quantity and quality of exercise for developing and maintaining cardiorespiratory, musculoskeletal, and neuromotor fitness in apparently healthy adults: guidance for prescribing exercise. Med Sci Sports Exerc, 43(7), 1334-1359. doi:10.1249/MSS.0b013e318213fefb

Gibala, M. J., Little, J. P., van Essen, M., Wilkin, G. P., Burgomaster, K. A., Safdar, A., . . . Tarnopolsky, M. A. (2006). Short-term sprint interval versus traditional endurance training: similar initial adaptations in human skeletal muscle and exercise performance. J Physiol, 575(Pt 3), 901-911. doi:10.1113/jphysiol.2006.112094

Gillen, J. B., Martin, B. J., MacInnis, M. J., Skelly, L. E., Tarnopolsky, M. A., \& Gibala, M. J. (2016). Twelve Weeks of Sprint Interval Training Improves Indices of Cardiometabolic Health Similar to Traditional Endurance Training despite a Five-Fold Lower Exercise Volume and Time Commitment. PLoS One, 11(4), e0154075. doi:10.1371/journal.pone.0154075

Gillen, J. B., Percival, M. E., Ludzki, A., Tarnopolsky, M. A., \& Gibala, M. J. (2013). Interval training in the fed or fasted state improves body composition and muscle oxidative capacity in overweight women. Obesity (Silver Spring), 21(11), 2249-2255. doi:10.1002/oby.20379

Gillen, J. B., Percival, M. E., Skelly, L. E., Martin, B. J., Tan, R. B., Tarnopolsky, M. A., \& Gibala, M. J. (2014). Three minutes of all-out intermittent exercise per week increases skeletal muscle oxidative capacity and improves cardiometabolic health. PLoS One, 9(11), e111489. doi:10.1371/journal.pone.0111489

Green, N., Wertz, T., LaPorta, Z., Mora, A., Serbas, J., \& Astorino, T. A. (2017). Comparison of Acute Physiological and Psychological Responses Between Moderate Intensity Continuous Exercise and three Regimes of High Intensity Training. J Strength Cond Res. doi:10.1519/JSC.0000000000002154

Hallal, P. C., Andersen, L. B., Bull, F. C., Guthold, R., Haskell, W., Ekelund, U., \& Group, L. P. A. S. W. (2012). Global physical activity levels: surveillance progress, pitfalls, and prospects. Lancet, 380(9838), 247-257. doi:10.1016/S0140-6736(12)60646-1

Hardcastle, S. J., Ray, H., Beale, L., \& Hagger, M. S. (2014). Why sprint interval training is inappropriate for a largely sedentary population. Front Psychol, 5, 1505. doi:10.3389/fpsyg.2014.01505

Hardy, C., \& Rejeski, W. (1989). Not what, but how one feels: the measurement of affect during exercise. Journal of Sport and Exercise Psychology, 11, 304-317.

Hargreaves, E., \& Stych, K. (2013). Exploring the peak and end rule of past affective episodes within the exercise context. Psychology of Sport and Exercise, 14(2), 169-178. doi:https://doi.org/10.1016/j.psychsport.2012.10.003 
Heisz, J. J., Tejada, M. G., Paolucci, E. M., \& Muir, C. (2016). Enjoyment for High-Intensity Interval Exercise Increases during the First Six Weeks of Training: Implications for Promoting Exercise Adherence in Sedentary Adults. PLoS One, 11(12), e0168534. doi:10.1371/journal.pone.0168534

Hopkins, W. G., Marshall, S. W., Batterham, A. M., \& Hanin, J. (2009). Progressive statistics for studies in sports medicine and exercise science. Med Sci Sports Exerc, 41(1), 3-13. doi:10.1249/MSS.0b013e31818cb278

Howell, D. (1997). Statistical Methods for Pyschology. Florence, Kentucky, U.S.A.: Brooks Cole.

Hu, L., Motl, R. W., McAuley, E., \& Konopack, J. F. (2007). Effects of self-efficacy on physical activity enjoyment in college-aged women. Int J Behav Med, 14(2), 92-96.

Jung, M. E., Bourne, J. E., \& Little, J. P. (2014). Where does HIT fit? An examination of the affective response to high-intensity intervals in comparison to continuous moderateand continuous vigorous-intensity exercise in the exercise intensity-affect continuum. PLoS One, 9(12), e114541. doi:10.1371/journal.pone.0114541

Kearon, M. C., Summers, E., Jones, N. L., Campbell, E. J., \& Killian, K. J. (1991). Effort and dyspnoea during work of varying intensity and duration. Eur Respir J, 4(8), 917-925.

Kendzierski, D., \& DeCarlo, K. J. (1991). Physical Activity Enjoyment Scale: Two Validation Studies. Journal of Sport and Exercise Psychology, 13(1), 50-64. doi:https://doi.org/10.1123/jsep.13.1.50

Kilpatrick, M. W., Martinez, N., Little, J. P., Jung, M. E., Jones, A. M., Price, N. W., \& Lende, D. H. (2015). Impact of high-intensity interval duration on perceived exertion. Med Sci Sports Exerc, 47(5), 1038-1045. doi:10.1249/MSS.0000000000000495

Korkiakangas, E. E., Alahuhta, M. A., \& Laitinen, J. H. (2009). Barriers to regular exercise among adults at high risk or diagnosed with type 2 diabetes: a systematic review. Health Promot Int, 24(4), 416-427. doi:10.1093/heapro/dap031

Little, J. P., Gillen, J. B., Percival, M. E., Safdar, A., Tarnopolsky, M. A., Punthakee, Z., . . . Gibala, M. J. (2011). Low-volume high-intensity interval training reduces hyperglycemia and increases muscle mitochondrial capacity in patients with type 2 diabetes. J Appl Physiol, 111(6), 1554-1560. doi:japplphysiol.00921.2011 [pii]

10.1152/japplphysiol.00921.2011

MacInnis, M. J., Zacharewicz, E., Martin, B. J., Haikalis, M. E., Skelly, L. E., Tarnopolsky, M. A., . . Gibala, M. J. (2017). Superior mitochondrial adaptations in human skeletal muscle after interval compared to continuous single-leg cycling matched for total work. J Physiol, 595(9), 2955-2968. doi:10.1113/JP272570 
Malik, A. A., Williams, C. A., Bond, B., Weston, K. L., \& Barker, A. R. (2017). Acute cardiorespiratory, perceptual and enjoyment responses to high-intensity interval exercise in adolescents. Eur J Sport Sci, 17(10), 1335-1342. doi:10.1080/17461391.2017.1364300

Martinez, N., Kilpatrick, M. W., Salomon, K., Jung, M. E., \& Little, J. P. (2015). Affective and Enjoyment Responses to High-Intensity Interval Training in Overweight-to-Obese and Insufficiently Active Adults. J Sport Exerc Psychol, 37(2), 138-149. doi:10.1123/jsep.2014-0212

Maxwell, S. E., \& Delaney, H. D. (2004). Designing experiments and analyzing data : a model comparison perspective (2nd ed. ed.). Mahwah, N.J. ; London: Lawrence Erlbaum Associates.

McAuley, E., \& Blissmer, B. (2000). Self-efficacy determinants and consequences of physical activity. Exerc Sport Sci Rev, 28(2), 85-88.

Metcalfe, R., Fawkner, S., \& Vollaard, N. (2016). No Acute Effect of Reduced-exertion Highintensity Interval Training (REHIT) on Insulin Sensitivity. Int J Sports Med. doi:10.1055/s-0035-1569450

Metcalfe, R. S., Babraj, J. A., Fawkner, S. G., \& Vollaard, N. B. (2012). Towards the minimal amount of exercise for improving metabolic health: beneficial effects of reducedexertion high-intensity interval training. Eur J Appl Physiol, 112(7), 2767-2775. doi:10.1007/s00421-011-2254-z

Metcalfe, R. S., Koumanov, F., Ruffino, J. S., Stokes, K. A., Holman, G. D., Thompson, D., \& Vollaard, N. B. (2015). Physiological and molecular responses to an acute bout of reduced-exertion high-intensity interval training (REHIT). Eur J Appl Physiol, 115(11), 2321-2334. doi:10.1007/s00421-015-3217-6

Metcalfe, R. S., Tardif, N., Thompson, D., \& Vollaard, N. B. (2016). Changes in aerobic capacity and glycaemic control in response to reduced-exertion high-intensity interval training (REHIT) are not different between sedentary men and women. Appl Physiol Nutr Metab, 41(11), 1117-1123. doi:10.1139/apnm-2016-0253

Metcalfe, R. S., Fitzpatrick, B., Fitzpatrick, S., McDermott, G., Brick N., McClean C \& Davison, G. W. (2016). Extremely short duration interval exercise improves 24-h glycaemia in men with type 2 diabetes Eur $J$ Appl Physiol. doi: https://doi.org/10.1007/s00421-018-3980-2

Nalçakan, G. R., Songsorn, P., Fitzpatrick, B. L., Yüzbasioglu, Y., Brick, N. E., Metcalfe, R. S., \& Vollaard, N. B. J. (2018). Decreasing sprint duration from 20 to $10 \mathrm{~s}$ during reduced-exertion high-intensity interval training (REHIT) attenuates the increase in maximal aerobic capacity but has no effect on affective and perceptual responses. Appl Physiol Nutr Metab, 43(4), 338-344. doi:10.1139/apnm-2017-0597 
Niven, A., Thow, J., Holroyd, J., Turner, A. P., \& Phillips, S. M. (2018). Comparison of affective responses during and after low volume high-intensity interval exercise, continuous moderate- and continuous high-intensity exercise in active, untrained, healthy males. J Sports Sci, 36(17), 1993-2001. doi:10.1080/02640414.2018.1430984

Reichert, F. F., Barros, A. J., Domingues, M. R., \& Hallal, P. C. (2007). The role of perceived personal barriers to engagement in leisure-time physical activity. Am J Public Health, 97(3), 515-519. doi:10.2105/AJPH.2005.070144

Rhodes, R. E., \& Kates, A. (2015). Can the Affective Response to Exercise Predict Future Motives and Physical Activity Behavior? A Systematic Review of Published Evidence. Ann Behav Med, 49(5), 715-731. doi:10.1007/s12160-015-9704-5

Ruffino, J. S., Songsorn, P., Haggett, M., Edmonds, D., Robinson, A. M., Thompson, D., \& Vollaard, N. B. (2017). A comparison of the health benefits of reduced-exertion highintensity interval training (REHIT) and moderate-intensity walking in type 2 diabetes patients. Appl Physiol Nutr Metab, 42(2), 202-208. doi:10.1139/apnm-2016-0497

Saanijoki, T., Nummenmaa, L., Koivumäki, M., Löyttyniemi, E., Kalliokoski, K. K., \& Hannukainen, J. C. (2018). Affective Adaptation to Repeated SIT and MICT Protocols in Insulin-Resistant Subjects. Med Sci Sports Exerc, 50(1), 18-27. doi:10.1249/MSS.0000000000001415

Stork, M. J., Banfield, L. E., Gibala, M. J., \& Martin Ginis, K. A. (2017). A scoping review of the psychological responses to interval exercise: is interval exercise a viable alternative to traditional exercise? Health Psychol Rev, 11(4), 324-344. doi:10.1080/17437199.2017.1326011

Stork, M. J., Gibala, M. J., \& Martin Ginis, K. A. (2018). Psychological and behavioral responses to interval and continuous exercise. Med Sci Sports Exerc. doi:10.1249/MSS.0000000000001671

Thomas, S., Reading, J., \& Shephard, R. J. (1992). Revision of the Physical Activity Readiness Questionnaire (PAR-Q). Can J Sport Sci, 17(4), 338-345.

Thum, J. S., Parsons, G., Whittle, T., \& Astorino, T. A. (2017). High-Intensity Interval Training Elicits Higher Enjoyment than Moderate Intensity Continuous Exercise. PLoS One, 12(1), e0166299. doi:10.1371/journal.pone.0166299

Tjønna, A. E., Lee, S. J., Rognmo, Ø., Stølen, T. O., Bye, A., Haram, P. M., . . W Wisløff, U. (2008). Aerobic interval training versus continuous moderate exercise as a treatment for the metabolic syndrome: a pilot study. Circulation, 118(4), 346-354. doi:10.1161/CIRCULATIONAHA.108.772822

Townsend, L. K., Islam, H., Dunn, E., Eys, M., Robertson-Wilson, J., \& Hazell, T. J. (2017). Modified sprint interval training protocols. Part II. Psychological responses. Appl Physiol Nutr Metab, 42(4), 347-353. doi:10.1139/apnm-2016-0479 
Vella, C. A., Taylor, K., \& Drummer, D. (2017). High-intensity interval and moderate-intensity continuous training elicit similar enjoyment and adherence levels in overweight and obese adults. Eur J Sport Sci, 17(9), 1203-1211. doi:10.1080/17461391.2017.1359679

Vollaard, N. B., \& Metcalfe, R. S. (2017). Research into the Health Benefits of Sprint Interval Training Should Focus on Protocols with Fewer and Shorter Sprints. Sports Med, 47(12), 2443-2451. doi:10.1007/s40279-017-0727-x

Vollaard, N. B. J., Metcalfe, R. S., \& Williams, S. (2017). Effect of Number of Sprints in an SIT Session on Change in V`O2max: A Meta-analysis. Med Sci Sports Exerc, 49(6), 1147-1156. doi:10.1249/MSS.0000000000001204

Williams, D.M., Dunsiger, S., Ciccolo, J.T., Lewis, B.S., Albrecht, A.E., \& Marcus, B.H. (2008). Acute affective response to a moderate-intensity exercise stimulus predicts physical activity participation 6 and 12 months later. Psychology of Sport and Exercise, 9(3), 231-254. https://doi.org/10.1016/j.psychsport.2007.04.002 

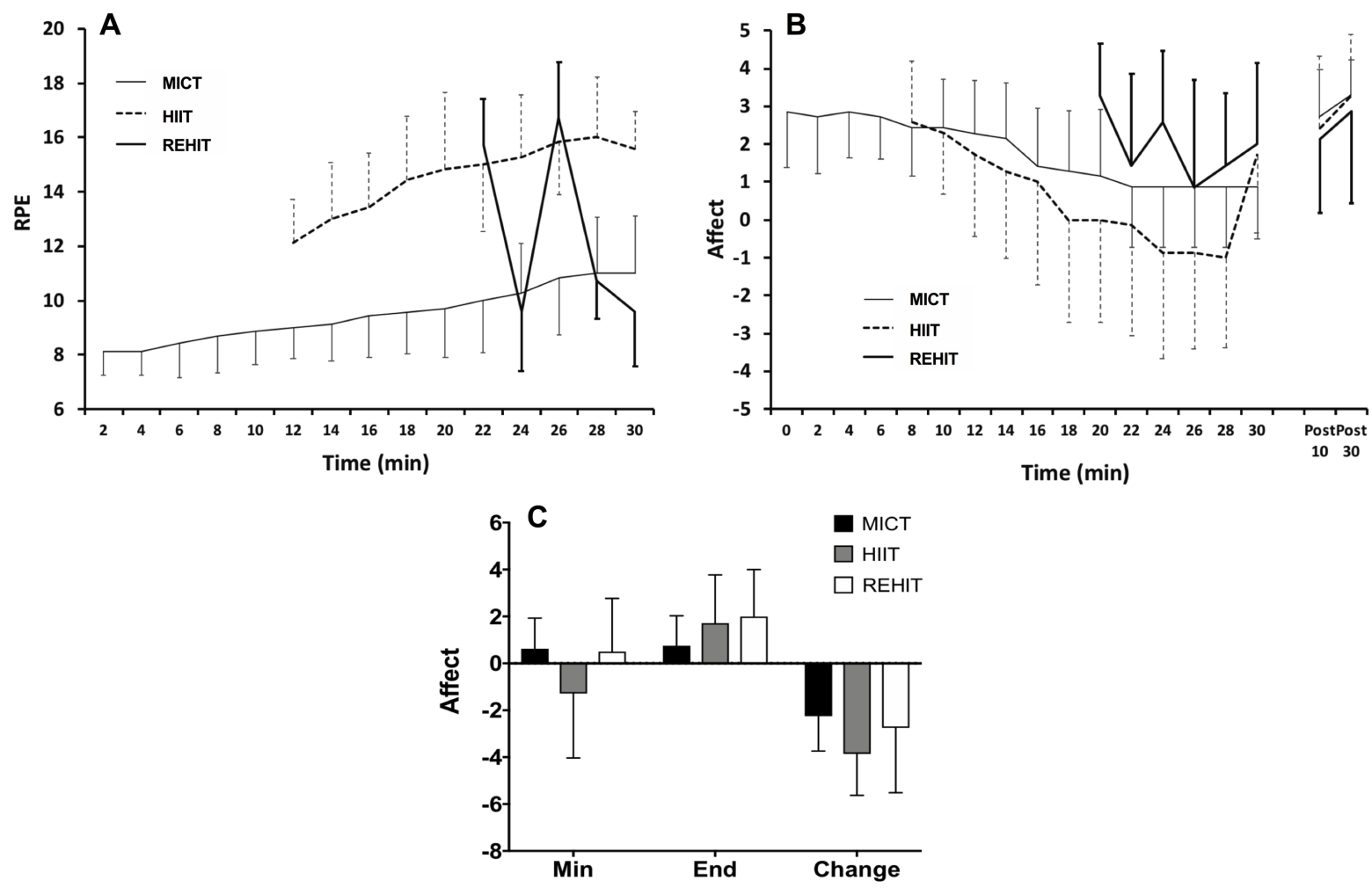

Figure 1 RPE and affective responses to REHIT, HIIT and MICT in healthy young participants (A and B) and comparisons of affective summary variables between exercise conditions (C). Data is shown as mean and SD for $\mathrm{n}=7$. 

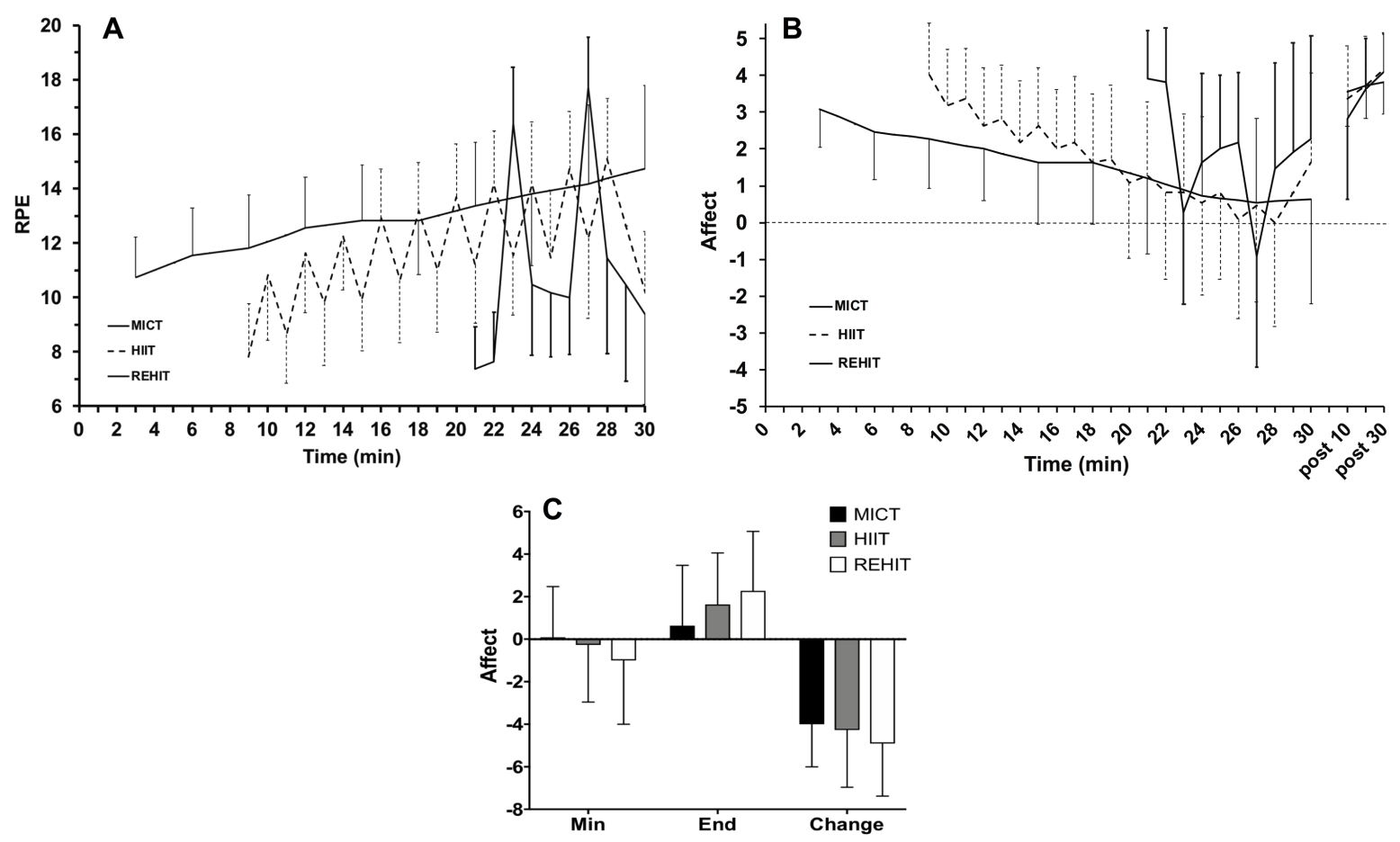

Figure 2 RPE and affective responses to REHIT, HIIT and MICT in men with type 2 diabetes (A and $\mathrm{B}$ ) and comparisons of affective summary variables between exercise conditions (C). Data is shown as mean and SD for $n=11$. 

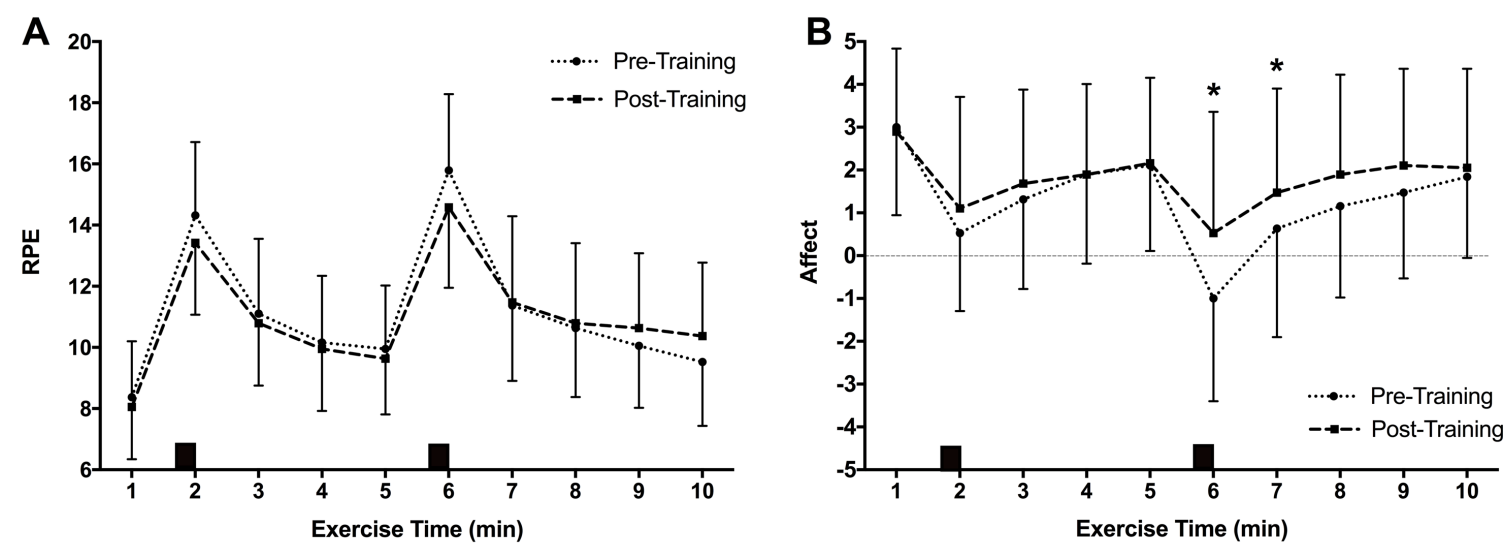

Figure 3 The effect of training on the acute RPE (A) and affective (B) responses to REHIT. Data presented and SD for $\mathrm{n}=19$. * denotes $p<0.05$ pre vs post. Filled boxes denote the timing of the all-out sprints. 